\title{
An Indigenous Dynamic Multiaxial Adjustable Shoulder Splint for the Management of Shoulder Subluxation
}

\author{
Surajit Kumar Sahu ${ }^{1}$, Minakshi Behera ${ }^{2}$, C. R. Mohanty ${ }^{3}$ \\ ${ }^{1}$ Ex-Assistant Professor (P\&O), ISHWAR Institute of Prosthetics \& Orthotics, AA-23, 3RD Street, 3RD Main \\ Road, Anna Nagar, Chennai-600040, Tamil Nadu \\ ${ }^{2}$ Assistant Professor (P\&O), National Institute for Empowerment of Persons with Multiple Disabilities \\ (Divyangjan), ECR, Muttukadu, Kovalam Post, Chennai-603112, Tamil Nadu \\ ${ }^{3}$ Retired Orthotist, Swami Vivekananda National Institute of Rehabilitation Training and Research, Olaptur, \\ Bairoi, Cuttack-754010, Odisha \\ Corresponding Author: Minakshi Behera
}

\begin{abstract}
Background: The main problem with shoulder subluxation is the instability of the shoulder joint. The anatomy of this joint permits a large range of movement, but it sacrifices stability. Available splint options for shoulder subluxations are a lot but they are not providing the full range of motion of the joint. Considering this we have designed and developed a new Dynamic Multiaxial Adjustable Shoulder Splint.

Objective: This study aimed to design an Indigenous Dynamic Multiaxial Adjustable Shoulder Splint for the Management of shoulder subluxation which will reduce the subluxation and pain.

Study design: A case report

Methods: A 52-years-old lady diagnosed with right shoulder subluxation was fitted with the newly designed Indigenous Dynamic Multiaxial Adjustable Shoulder Splint for the reduction of pain and subluxation. The Western Ontario Shoulder Instability Index (WOSI) was assessed to measure the shoulder-related quality of life in patients with symptomatic shoulder instability. Before and after the test was conducted with and without the newly designed splint with an intervention period of 4 months.

Results: The WOSI score of $9.8 \%$ was achieved after the intervention period of 4 months and the reduction of subluxation was immediate and provided total relief from the pain.

Conclusion: This Indigenous Dynamic Multiaxial Adjustable Shoulder Splint can be considered as a very excellent splint for the orthotic management of shoulder subluxation and it also can be used as a standard design that can be prescribed for patients with brachial plexus injury, frozen shoulder and rotator cuff injury, post-operative cases, etc.
\end{abstract}

Key Words: Shoulder Subluxation, Multiaxial, shoulder splint, WOSI score.

\section{INTRODUCTION}

As a consequence of a brachial plexus lesion or hemiplegia, a subluxation of the shoulder frequently occurs. Shoulder subluxation is defined as a partial or incomplete dislocation that usually stems from changes in the mechanical integrity of the joint. In subluxation, the humeral head slips out of the glenoid cavity as a result of weakness in the rotator cuff or a blow to the shoulder area. A subluxation can occur in one of three types: anterior (forward), posterior (backward), and inferior (downward).

Only one out of five hemiplegic patients end up having any use of the upper limb, frequently because of loss of shoulder function. The function of the shoulder joint 
is to precisely position the arm in space to allow self-care and manipulation of the environment by the forearm and hand ${ }^{(1)}$.

The subluxation of the shoulder in hemiplegia is due to a decrease of cerebral control over the patterned and coordinated interaction of muscles controlling the position and movement of the scapula and humerus, with the result that spastic muscles tilt the scapula laterally and paretic muscles contribute to unseating of the humeral head from the glenoid fossa. Pain and reflexive spread of spasticity invariably result from attempted movement, and the recovery of shoulder function lags behind that of the elbow and wrist ${ }^{(2)}$.

Smith and Okamoto (1981) have described several designs of orthoses for the subluxed shoulder, together with a checklist for hemiplegic slings.

Most patients, therefore, are advised to wear a shoulder sling, which, does not reduce the subluxation nor relieve pain ${ }^{(3)}$. Given the current state of knowledge, the present study aimed to design an Indigenous Dynamic Multiaxial Adjustable Shoulder Splint for the management of Shoulder Subluxation which will reduce the subluxation and pain.

\section{METHODS \\ Subject}

The patient was a 52-years-old lady who had right-side shoulder subluxation. She was a housewife and was using the conventional shoulder sling for the subluxated shoulder. She was referred to the Department of Orthotics and Prosthetics in the Swami Vivekananda National Institute of Training and Research (SVNIRTAR), for the provision of a suitable Dynamic Multiaxial Adjustable Shoulder Splint. The patient gave a written informed consent form to participate in the study, and appropriate approval was also obtained from the Institutional Ethical committee. A detailed assessment was performed with demographic data, medical history, radiographic image, and functional outcome.

\section{A New Design}

The new design is made of modular parts, which interact dynamically with each other. This Orthosis is made up of seven components, pelvic section, lateral aluminium upright, multiaxial shoulder joint, elbow joint section with arm and forearm upright, arm and forearm shell, scapular stabilization component, Elastic webbing of 2 inches, and thoracic support.

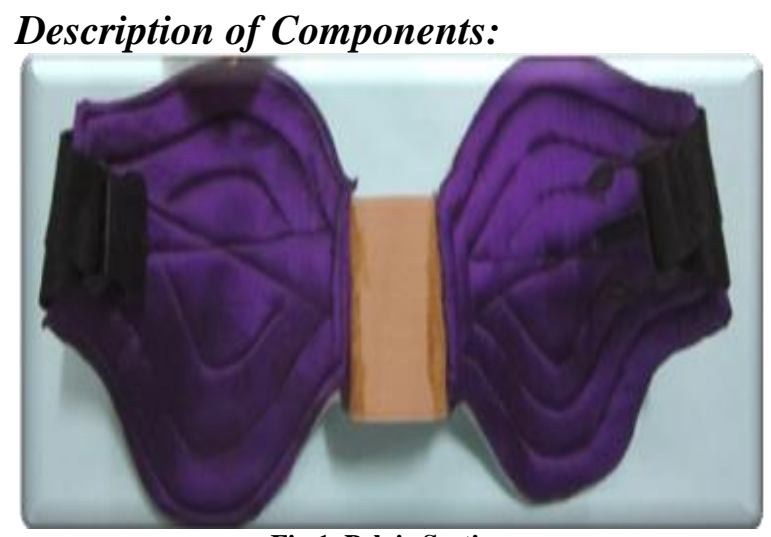

Fig 1. Pelvic Section

The Pelvic component was specially designed for total bearing the weight of the orthosis as well as stabilizing the orthosis with the patient by one nylon webbing attached with the posterior side to connect both the pelvic component and one adjustable strap with buckle attached with anterior side. The pelvic component is made up of a $4 \mathrm{~mm}$ non-toxic polypropylene sheet for better cosmetic purposes and light in weight.

Lateral uprights made up of aluminium alloy with a length of $30 \mathrm{~mm}$ and thickness of $4 \mathrm{~mm}$, distally connected with pelvic components and proximally attached with multiaxial shoulder joint.

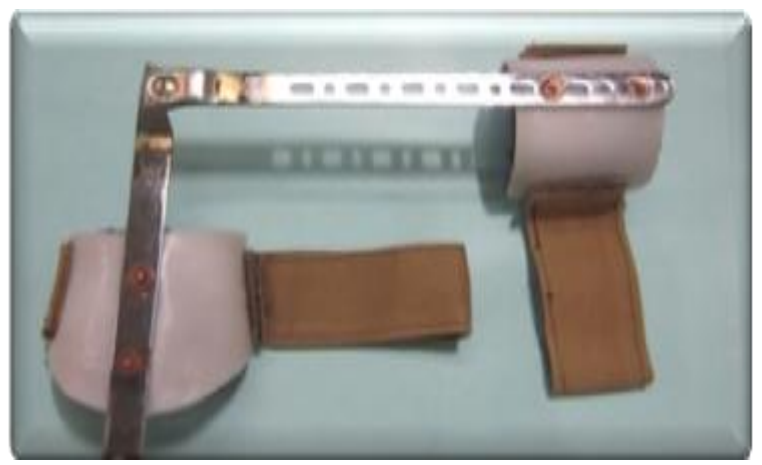

Fig 2. Multiaxial Shoulder Joint 


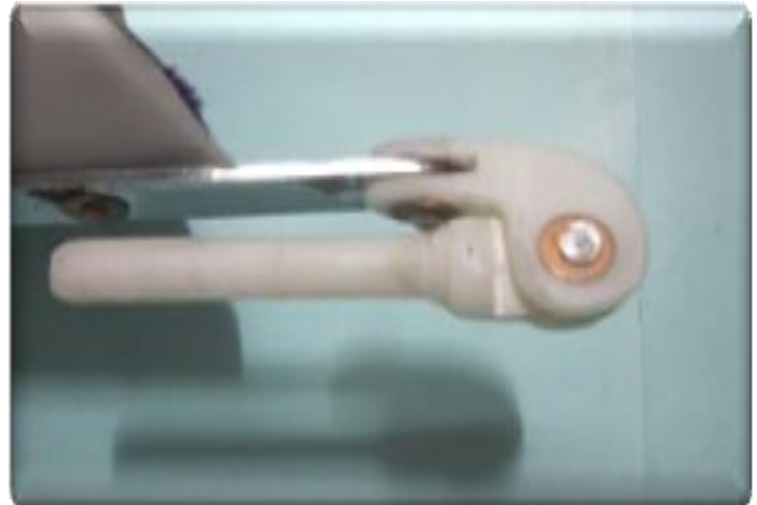

Fig 3. Elbow joint with humeral and forearm cuff

The multiaxial shoulder joint is specially designed to allow flexion \& extension, abduction \& adduction, and rotation of the shoulder and it is offering a true choice of position and optional scapular stabilization that limits anterior movements of the scapula. It helps in their ADL activities and permits friction-free movement.

A pair of cuffs, humeral and forearm, attach to the limb above and below the elbow joint, respectively. The elbow joint provides a full range of flexion and extension movement.

The optional scapula stabilization component limits anterior movement of the scapula allowing connective tissue between the shoulder and the scapula to be stretched. Thoracic support is provided for the attachment of the scapular stabilization component and webbing straps.

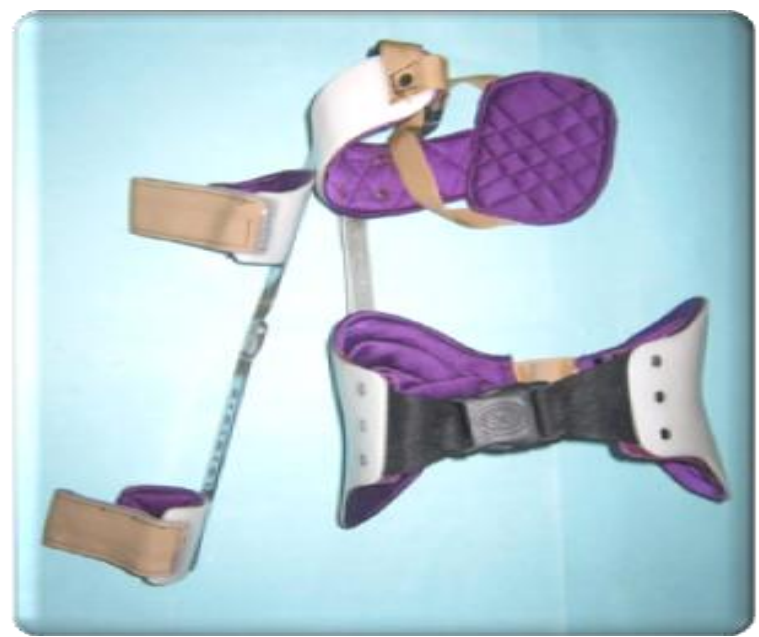

Fig 4. Indigenous Dynamic Multiaxial Adjustable Shoulder Splint

\section{Fabrication Procedure:}

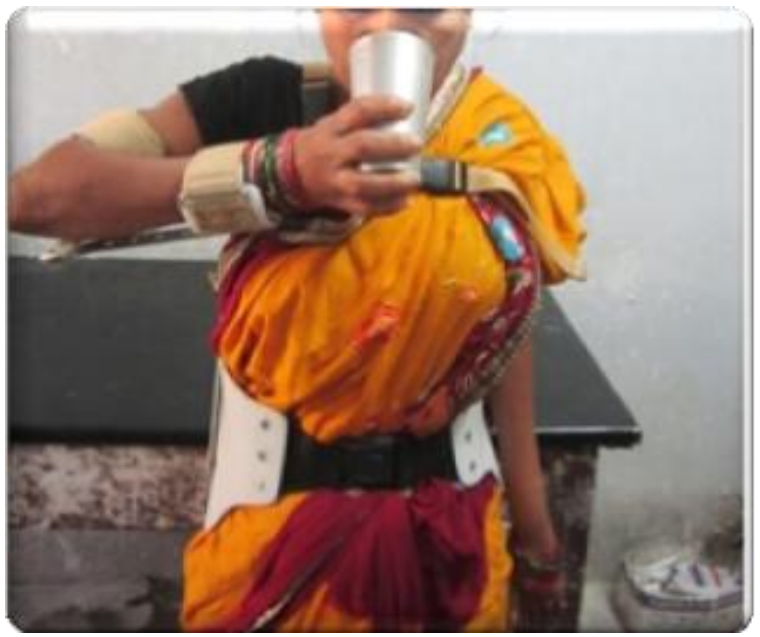

Fig 5. Indigenous Dynamic Multiaxial Adjustable Shoulder Splint with the Patient

Two pelvic girdles are attached by an elastic webbing (2 inches width) Posteriorly. Then laterally adjustable aluminium upright (1 $1 / 2$ inch width) is attached with the right pelvic girdle with the help of two $5 \mathrm{~mm}$ screws. With that upright a multiaxial shoulder joint is attached which provides flexion \& extension, abduction \& adduction, and also rotation. Stainless steel upright of the arm section is hinged with the shoulder joint and another upright is attached for the forearm section on the other side of the elbow joint. A size-I hip joint of ALIMCO made, which was modified as an elbow joint that provides a full range of flexion \& extension. In arm and forearm uprights, arm and forearm shells were attached respectively. Thoracic support is attached with the lateral aluminium upright. Then scapular stabilization component is attached with the lateral aluminium upright. After all this, a $1 \frac{1}{2}$ inches webbing strap with an adjustable lock is attached with both the pelvic girdles by press buttons. Then one over shoulder strap with an adjustable lock is attached with both sides of the thoracic support component. Another auxiliary strap with an adjustable lock is attached with both the shoulder stabilization component coming under the axilla joined over the chest. 


\section{RESULTS}

The WOSI score of $24.4 \%$ was achieved after the use of this Indigenous Dynamic Multiaxial Adjustable Shoulder Splint which was $83.3 \%$ before using the splint. The Western Ontario Shoulder Instability Index (WOSI) is a 21-item scale used to measure the shoulder-related quality of life in patients with symptomatic shoulder instability.
With the Indigenous Dynamic Multiaxial Adjustable Shoulder Splint, the reduction of subluxation was immediate and provided relief from pain. After 4 months of follow-up, the WOSI score of $9.8 \%$ was achieved which shown the great effectiveness of this newly designed Indigenous Dynamic Multiaxial Adjustable Shoulder Splint.

Table 1: Result of the WOSI Score

\begin{tabular}{|l|l|l|l|}
\hline Outcome Measure & Score Before the Intervention & Score Immediate after the Intervention & Score After 4-months of Follow-up \\
\hline WOSI Score & $83.3 \%$ & $24.4 \%$ & $9.8 \%$ \\
\hline
\end{tabular}

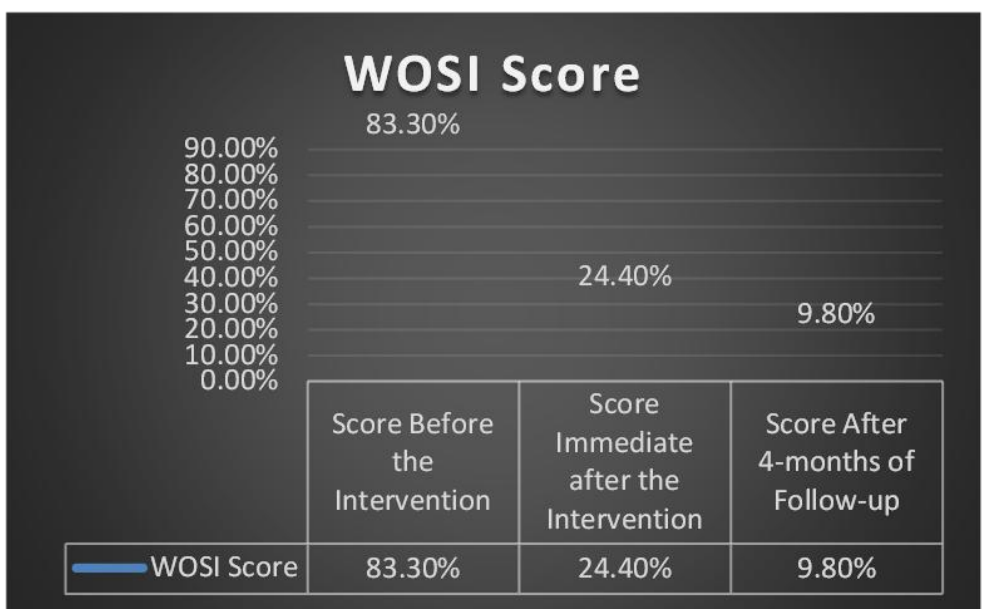

\section{DISCUSSION}

Loss of shoulder function is of serious consequences in any illness or injury and limits the use of the entire limb, resulting in more often than not in a permanent disability of the afflicted individual $^{(4)}$. The splint can, therefore, also be a valuable aid where traumatic, inflammatory, and degenerative diseases have affected the shoulder joint and temporarily compromised its crucial function of self-care and control of the environment. The splint can be used in the conservative treatment of the postsurgical phase of fractures of humeral neck and head, rotator cuff tears, and posterior shoulder dislocations; in incomplete brachial plexus lesions (C5, C6); in frozen shoulder and shoulder/hand syndromes; and in the post-surgical phase of the total shoulder joint replacement. With the shoulder securely stabilized and the arm kept in a physiological position of function, the forearm and hand can be used functionally during the often lengthy period of the needed shoulder immobilization.

This orthosis acts as dynamic orthosis and can be prescribed to stabilize and control rotation in the shoulder when the elbow flexors and wrist extensors have recovered and the shoulder muscles have recovered partially. The orthosis helps in shoulder stabilization and rotation by using the residual muscle power being assisted by the control straps which the orthosis also prevents shoulder subluxation. The disadvantage of this splint is that the chest strap creates irritation in the female client. Although the satisfactory result was achieved in this case with the Indigenous Dynamic Multiaxial Adjustable Shoulder Splint, further investigation is needed to prove the consistency of the result.

Future research should identify more populations in a variety of healthcare systems that may benefit from an 
Indigenous Dynamic Multiaxial Adjustable Shoulder Splint.

\section{CONCLUSION}

It can be concluded that shoulder subluxation deformity can be effectively treated by this Indigenous Dynamic Multiaxial Adjustable Shoulder Splint. The introduction of the Indigenous Dynamic Multiaxial Adjustable Shoulder Splint has opened up a vast area of new orthotic input towards a more meaningful rehabilitation of the shoulder subluxation. This orthosis is simple, effective, light in weight, inexpensive, and the most important thing is that it can be used in any kind of shoulder subluxation. It is a standard design that can be prescribed for patients with brachial plexus injury, frozen shoulder and rotator cuff injury, post-operative cases, subluxation, etc.

Conflict of Interest: The author does not have any conflict of interest regarding research, authorship, and publication of this article.

Source of Funding: The research receives no specific grant from any specific agency in the public, commercial or non-profit sectors. The authors declare that no competing interests exist.

Authors' Contributions: The entire clinical course of An Indigenous Dynamic Multiaxial Adjustable Shoulder Splint service delivery was done by Mr. Surajit Kumar Sahu towards the fulfillment of a bachelor's degree research project under the guidance of Mr. C. R. Mohanty. The manuscript preparation is done by Mrs. Minakshi Behera and Mr. Surajit Kumar Sahu. All the clinical service delivery to patients and research study was carried out in the premises of SVNIRTAR, Cuttack.

\section{ACKNOWLEDGMENTS}

We would like to extend our gratitude to Mr. S. N. Rout, former HoD of Prosthetic \& Orthotic Department, SVNIRTAR, Olatpur for providing all the necessary types of equipment and infrastructure during the research study. The authors are thankful to all the staff of the Prosthetic and Orthotic Department of SVNIRTAR for providing the necessary support.

\section{REFERENCES}

1. Codman, E.A., The Shoulder, Thomas Todd, Boston, 1936.

2. Caillet, R., The Shoulder in Hemiplegia, F.A. Davis, Philadelphia, 1980.

3. Hurd, M. "Shoulder sling for hemiplegia: Friend or foe?" Arch. Phys. Med. Rehabil., 1975, 55:519-522.

4. U.S. Dept. of Labor, International Assoc. of Compensation Boards: Bulletin, 167, 1952, Washington, D.C.

How to cite this article: Sahu SK, Behera M, Mohanty CR. An indigenous dynamic multiaxial adjustable shoulder splint for the management of shoulder subluxation. Int $J$ Health Sci Res. 2021; 11(6): 140-144. DOI: https://doi.org/10.52403/ijhsr.20210620 